\title{
WearTrack - Tracking Valuables using Near Field Communication Stickers
}

\author{
G. Muneeswari ${ }^{1 *}$, J. Frank Vijay ${ }^{2}$ \\ ${ }^{I}$ Professor, Department of Computer Science and Engineering, Vel Tech Multitech Dr.RR Dr.SR Engineering College \\ ${ }^{2}$ Professor \& HOD, Department of Information Technology, K.C.G. College of Technology \\ *Corresponding Author E-mail: muneeswarig@veltechmultitech.org
}

\begin{abstract}
In modern era, people will not be satisfied with technology until it not only reminds us of our day to day work but also does it for us without draining much of our energy. Any person will acknowledge the fact that we need smarter technology that reminds us what things are to be taken wherever we go or whenever we leave a place. It makes life so much easier if we are told to do things rather than thinking up things to be done on our own.People irrespective of their age, have always been in situations where they have lost their valuable things or have the fear of losing them.So in this paper an attempt is made how a user gets alarmed whenever they leave a place forgetting to take their valuables. The user has a sensorand othersensorsare connected to things which they want to forget to take as they leave a place. All the sensing devices consist of Bluetooth to recognize the user's sensor or to be recognized by it. An alarm is connected in the circuit which switches on whenever they leave the place without the required things. The evaluation results show that the sensors will give $98 \%$ accuracy towards the complete Bluetooth connection.
\end{abstract}

Keywords: WearTrack; Bluetooth; Near Field Communication; Trakkies; Pebblebee

\section{Introduction}

The paper aims at making peoples' lives easier by making sure people do not lose their valuables and not forget them when they leave a place. For example, a person leaving from home with all the valuables will not want to misplace any item. We have come up with a solution, which integrates all these accessories and manages to keep them connected virtually.Such integrated object allows us to keep track of all the items as we move around.With such a technology, the user will obviously come out of the fear of losing things wherever he goes. It is to help people make better use of technology in everyday life in such a fast-paced world.Some of the tracking devicesoffers a 200ft range tracker (Fig.1) which is a disadvantage since it will not be able to notify the user immediately and will respond late. For example, a person sitting at a coffee shop and assume that all the accessories are currently connected using tracking device. Now when the person decides to leave and forget to take the wallet which is a part of the network.

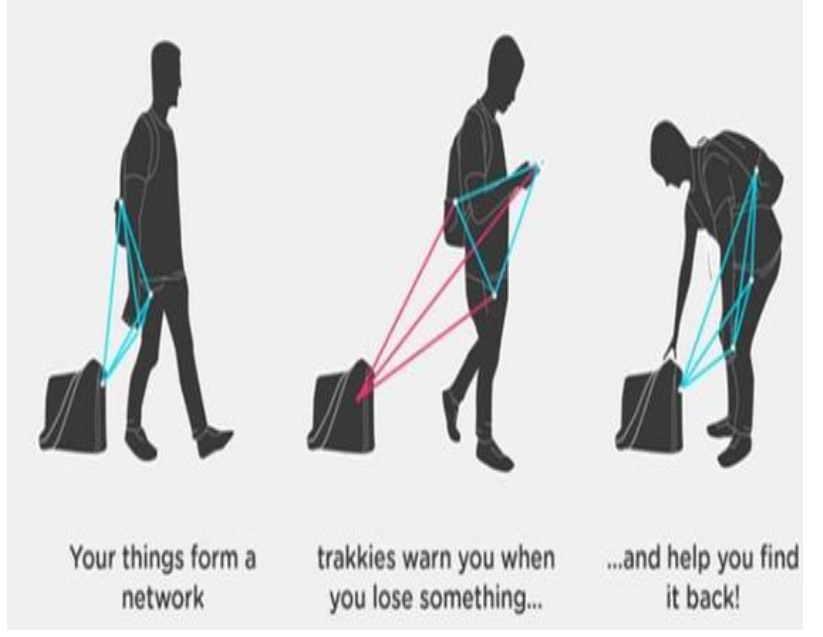

Fig. 1: Tracking Device - Working Methodology

The device will notify only after the person cross the $200 \mathrm{ft}$ range which makes it inconvenient for them to retrieve the wallet from the coffee shop. This isseem to be a major disadvantage which has been overcome in the proposed paper.

In this paper, section II discusses the literature review and section III describes the design of wear track device.Section IV explains about the results obtained and section $\mathrm{V}$ concludes the paper. 


\section{Literature Review}

The method will need to handle a large number of movingobjects at once.To address the scalability goals, we usehierarchical organization, similar to schemes previously used

in cellular telephone systems [4].A popular mobility modelused for several ad hoc network protocols $[5,6]$ consists ofnodes that move uniformly across a region under study, inorder to approximate small-scale scenarios where the movementsare expected to be random and close touniform.Pebblebee [2] is a small and sleek device operated with the help of a mobile app. It makes use of Bluetooth tracking technology to identify the various virtually connected accessories. It helps find any lost or misplaced item in the vicinity of the particular mobile device.

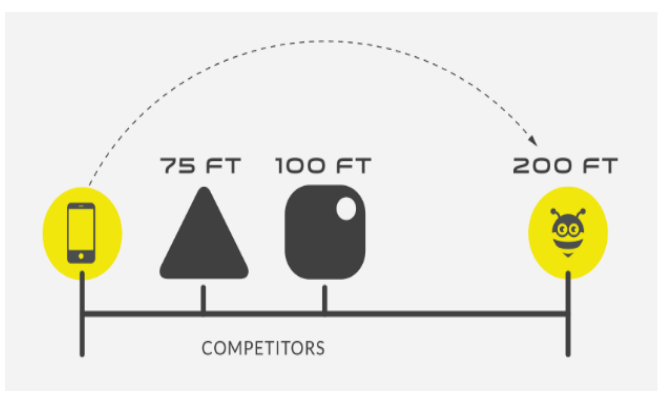

Fig. 2: Range supported Pebblebee [2]

Pebblebee is a long range tracker (Fig.2) and this product comes in three parts such as the finder, honey and stone.But there are several disadvantages with this product such as it requires a mobile app to be operated and cannot be used otherwise. It has three separate working units that have to be handled by the user effectively which makes it a tedious process. This product operates using Bluetooth technology which limits its application considerably.

Trakkies (Fig.3) [3] always form a smart feel around us that operate and function, creating an interface between the physical to digital worlds. It enables people, places and objects through an integrated platform, to connect to the world around them. This hardware has certain advanced technologies that helps keep track of our valuables.

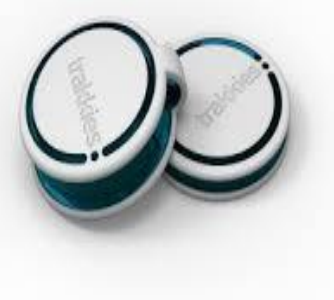

Fig. 3: Trakkies [3]

The proposed model completely overcomes these difficulties. It allows us to use it with ease and is much more simple and comfortable in terms of handling and usage. This is done by using stickers in place of the tags which are used in trakkies. It is much easier to use the stickers on all kinds of accessories in comparison to the heavy tags provided in the above application.[1]

\section{Design of WearTrack}

Our product has been designed using Near Field Communication (NFC) stickers. These NFC stickers are connected virtually using an android mobile application which has been developed exclusively for this purpose.

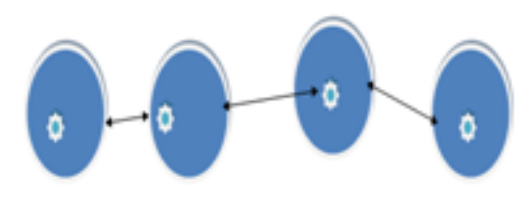

Fig. 4: Devices connected in the virtual network

The mobile application is used to keep track of the various accessories each of which have an NFC sticker that are uniquely identified with NFC ids. The application has been programmed to trigger an alarm in case one of the virtually connected devices leaves the network.
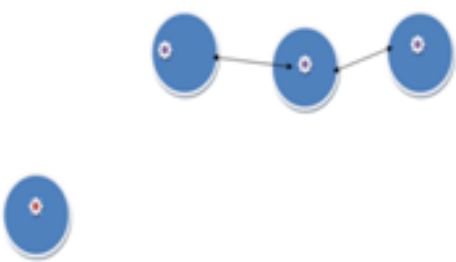

Fig. 5: Device leaving the network

In Fig.4, we see that all devices are connected together and in Fig.5, if a single device leaves the network it triggers an alarm in the user's mobile. This is the key characteristic property of the application. The application stores all the ids in an array. This array is scanned periodically for its contents. It ensures that no id is missing from the array. In case an id goes missing from the array list, the program triggers an alarm. Once found, the id is replaced or reconnected to the array list which completes the network again.

\section{Results and Discussion}

Since this application uses stickers for identification of accessories, it proves to be the lightest and most efficient in comparison to all the other contemporary systems of similar functionality.

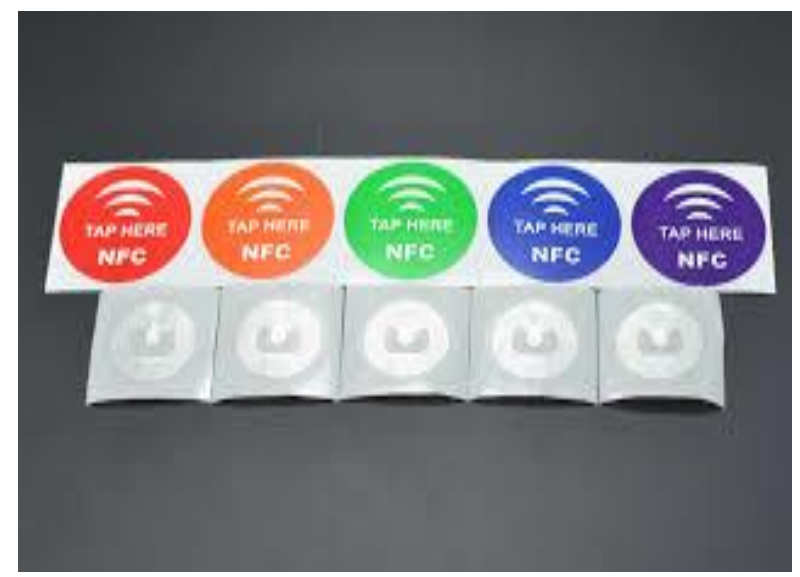

Fig. 6: NFC Stickers [5]

The NFC stickers (Fig.6) are the cheapest hardware for this purpose.It is also easily available in most of the E-commerce websites. The product can support a network that can include a large number of connected items and can also be expanded easily.It supports a small range of 60 meters which can be expanded based on user requirement specifications. For example, you may require the application to work in a relatively smaller cabin than a friend of yours who needs it running in a much larger room. In such scenarios, the app can be modified to use varying ranges. 
The proposed work has been implemented with a raspberry pi wherein the first step is to connect the card reader component to the raspberry pi. Then thesecond step is to connect raspberry piwith the system using a lan cable. Finally it could be switched on using an external USB cable. In order to configure the Raspberry Pi, PuTTY (Fig.7) which is a user interface used as a screen to communicate with the Raspberry Pi is downloaded.

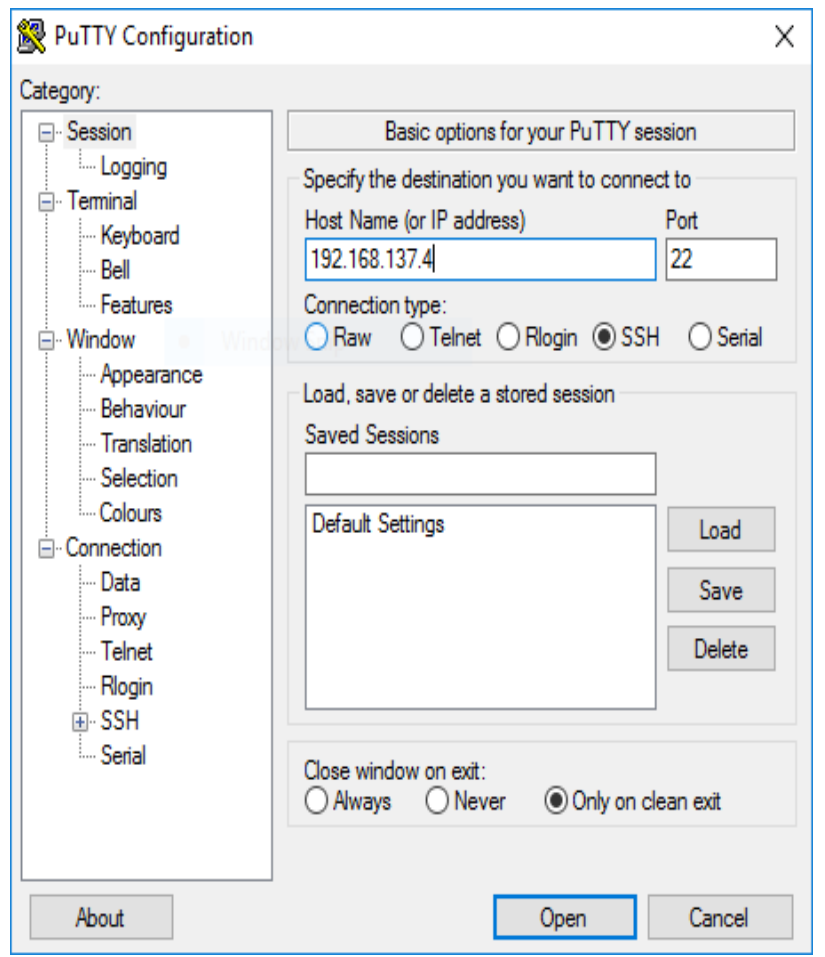

Fig.7: PuTTY Configuration

Then the Virtual Network Computing (VNC) is downloaded which is a graphical desktop sharing system that uses the Remote Frame Buffer protocol (RFB) to remotely control another computer. It transmits the keyboard and mouse events from one computer to another.PuTTY configuration can be set up with a Host Name or IP address: 192.168.137.4The authentication of the user is shown in Fig.8.

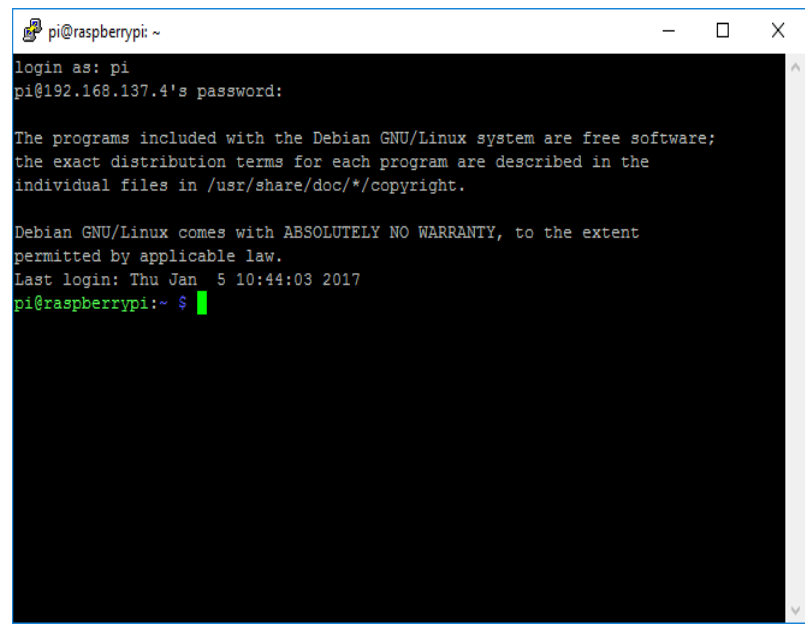

Fig. 8: Command Prompt authentication

After the user authentication, the device will be detected by the card reader and connected to the network.

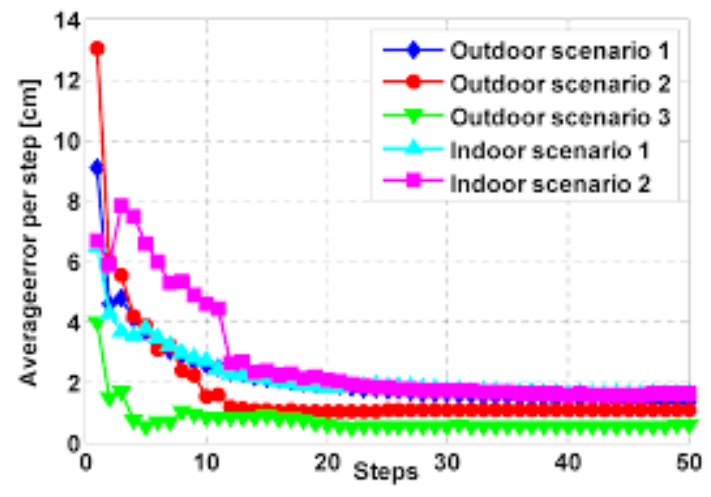

Fig. 9: Average error per step

In Fig.9, the average error per step for the indoor and outdoor scenarios of the sensor datais shown.The error rate is very less compared to the existing methods. The proposed system shows $98 \%$ accuracy for the indoor scenarios and it is expected to improve for large area network.

\section{Conclusion}

In a fast-paced world, such as this, where growth is exponential, technological advancements have always been welcomed and helpful. With the increase in dependence on devices and lack of patience in managing them, we rely on such solutions to device management. People of all age groups insist on a better system to secure their properties. Our application not only safeguards our items, but also offers the most affordable product when compared to its competitors. Remaining constantly in touch with our belongings, provides a psychological satisfaction to the user at all times who worries about losing things otherwise.

\section{References}

[1] https://www.kickstarter.com/projects/trakkies

[2] https://pebblebee.com/finder/

[3] https://www.trakkies.com/

[4] Pitoura, E. and Samaras, G., "Locating Objects in Mobile Computing,'IEEE Transactions on Knowledge and Data Engineering, Vol. 13, No.4, pages 571-592, July/August 2001

[5] Perkins, C. E. and Royer, E. M., "Ad hoc On-Demand Distance VectorRouting," Proc. 2nd IEEE Workshop on Mobile Computing Systemsand Applications, New Orleans, LA, February 2014.

[6] Pottie, G. J., and Kaiser, W. J. "Wireless Integrated Network Sensors,"in Communications of the ACM, Vol. 43, No. 5, May 2000.

[7] S.V.Manikanthan and K.srividhya "An Android based secure access control using ARM and cloud computing", Published in: Electronics and Communication Systems (ICECS), 2015 2nd International Conference on 26-27 Feb. 2015,Publisher: IEEE,DOI: 10.1109/ECS.2015.7124833.

[8] S.V.Manikanthan and T.Padmapriya "Recent Trends In $\mathrm{M} 2 \mathrm{~m}$ Communications In 4g Networks And Evolution Towards 5g", International Journal of Pure and Applied Mathematics, ISSN NO:1314-3395, Vol-115, Issue -8, Sep 2017.

[9] T.Padmapriya, S.V.Manikanthan, "An enhanced distributed evolved node-b architecture in 5G tele-communications network", International Journal of Engineering \& Technology, DOI: 10.14419/ijet.v7i2.8.10419, ISSN NO:2227-524X, Vol-7, No.2.9(2018) 